\title{
BMJ Open Manual Therapy for Fibrosis-Related Late Effect Dysphagia in head and neck cancer survivors: the pilot MANTLE trial
}

\author{
Katherine Hutcheson (D , ${ }^{1}$ Holly McMillan, ${ }^{2}$ Carla Warneke ${ }^{3}$ Christine Porsche, ${ }^{2}$ \\ Kiara Savage, ${ }^{2}$ Sheila Buoy, ${ }^{2}$ Jihong Wang, ${ }^{4}$ Karin Woodman, ${ }^{5}$ Stephen Lai, ${ }^{1}$ \\ Clifton Fuller ${ }^{6}$
}

To cite: Hutcheson $\mathrm{K}$, McMillan $\mathrm{H}$, Warneke C, et al. Manual Therapy for Fibrosis-Related Late Effect Dysphagia in head and neck cancer survivors: the pilot MANTLE trial. BMJ Open 2021;11:e047830. doi:10.1136/ bmjopen-2020-047830

- Prepublication history for this paper is available online. To view these files, please visit the journal online (http://dx.doi. org/10.1136/bmjopen-2020047830).

Received 10 December 2020 Accepted 17 July 2021

Check for updates

(c) Author(s) (or their employer(s)) 2021. Re-use permitted under CC BY-NC. No commercial re-use. See rights and permissions. Published by BMJ.

For numbered affiliations see end of article.

Correspondence to Dr Katherine Hutcheson; karnold@mdanderson.org

\section{ABSTRACT}

Introduction Late dysphagia that develops or persists years after head and neck cancer (HNC) is a disabling survivorship issue. Fibrosis is thought to stiffen connective tissues and compress peripheral nerve tracts, thereby contributing to diminished strength, flexibility, and in some cases denervation of swallowing muscles. Manual therapy (MT) is used in cancer survivors for pain and other indications, but it is unknown if increasing blood flow, flexibility and cervical range of motion (CROM) in the head and neck may improve late dysphagia.

Methods and analysis Manual Therapy for FibrosisRelated Late Effect Dysphagia (MANTLE) is a National Cancer Institute-funded prospective single-arm pilot trial evaluating the feasibility, safety and therapeutic potential of MT in patients with late dysphagia after radiotherapy (RT) for HNC. Disease-free survivors $\geq 2$ years after curative-intent RT for HNC with at least moderate dysphagia and $\geq 2$ Common Terminology Criteria for Adverse Events version 4.0 fibrosis are eligible. The target sample size is 24 participants who begin the MANTLE programme. MANTLE is delivered in 10 MT sessions over 6 weeks with an accompanying home exercise programme (HEP). Patients then transition to a 6-week post-washout period during which they complete the HEP and then return for a final post-washout evaluation. Feasibility (primary endpoint) and safety will be examined. Serial assessments include CROM, modified barium swallow studies, quantitative MRI, electromyography (optional) and patient-reported outcomes as secondary, tertiary and exploratory endpoints.

Ethics and dissemination The research protocol and informed consent document was approved by the Institutional Review Board at the University of Texas MD Anderson Cancer Center. Findings will be disseminated through peer-reviewed publication that will be made publicly available on PubMed Central on acceptance for publication, in compliance with NIH public access policy. Trial registration number NCT03612531.

\section{INTRODUCTION}

Dysphagia is a priority issue for head and neck cancer (HNC) survivors. While noteworthy as a driver of quality of life (QOL), ${ }^{1}$
Strengths and limitations of this study

- Manual Therapy for Fibrosis-Related Late Effect Dysphagia is a pilot, single-arm feasibility trial of manual therapy for late radiation-associated dysphagia with strengths including a diverse panel of secondary endpoints examining functional, physical and patient-reported outcomes.

- Strengths include examination of a novel therapy for an often refractory condition with comprehensive outcome measures.

- Limitations include pilot nature of the trial without a control group or lead-in and lack of cervical posture measures.

chronic, persistent or late dysphagia is also a serious health problem in long-term survivorship. Even in modern practice, chronic aspiration (airway entry of liquids or food) is a life-threatening manifestation of dysphagia afflicting up to $30 \%$ of survivors treated with definitive radiotherapy (RT) or chemoradiotherapy (CRT). ${ }^{2}$ HNC survivors treated with CRT are 2.7 times more likely to develop aspiration pneumonia than non-cancer controls, and aspiration pneumonia confers a $42 \%$ increased risk of mortality among survivors. ${ }^{3}$

There is a rapidly growing pool of $\mathrm{HNC}$ survivors at risk for late dysphagia. Almost half of HNCs are now human papillomavirus (HPV)-driven oropharyngeal cancers, the incidence of which is expected to increase through at least $2030 .{ }^{4}$ The vast majority of this fast-growing, large subgroup of HNC survivors has been treated with curative RT at doses of 60 Gray (Gy) or more to the pharyngeal axis sufficient to induce chronic or late radiation-associated dysphagia (RAD).$^{5-7}$ Distinct from tobacco-related HNC, HPVassociated $\mathrm{HNC}$ is diagnosed younger (median: 54 years) ${ }^{8}$ with excellent 2-year and 
5-year survival probability of $95 \%{ }^{9}$ and $79 \%,{ }^{10}$ respectively. For these reasons, modern HNC survivors with HPV-attributable oropharyngeal cancer have the potential to live many active years (even decades) with toxicities of RT.

While many survivors initially recover functional swallowing after acute effects of radiation resolve, an important subset develops debilitating persistent or late RAD. It is estimated $30 \%-40 \%$ treated with current treatment regimens that prescribe 66-72 Gy radiotherapy develop chronic RAD, and a highly burdened subset progress significantly over subsequent years. ${ }^{711}$

Late-RAD ${ }^{12-14}$ is a particularly challenging, typically progressive form of refractory dysphagia that manifests years into survivorship (median latency: 8 years, cumulative incidence $8 \%$ at 7 years survival) after a period of reasonable functional recovery. ${ }^{15}$ As the era of HPVassociated oropharyngeal cancer survivorship matures, the number of late-RAD cases grows. Late-RAD is among the most difficult late-effect conditions to manage in HNC survivors, associated with a cascade of functional decline. Late lower cranial neuropathies are highly prevalent in survivors with late-RAD, $48 \%-83 \%$ in the investigators' prior work, and increase the likelihood of lifelong feeding tube dependence due to refractory aspiration. ${ }^{51216}$ Despite standard therapies such as swallowing exercises with or without cervical oesophageal dilation, $66 \%$ of late-RAD cases in the investigators' published case series became chronically feeding tube dependent in late survivorship at a median age of 64 years (9 years after cancer cure). ${ }^{14}$ The QOL and health implications of becoming feeding tube dependent for life at this active age are staggering. ${ }^{14}$ Recent work highlights the gravity of this among patients with cancer who ranked feeding tube dependence as one of the top six outcomes of their cancer that they perceive to be worse than death. ${ }^{17}$

Therapy success in survivors with RAD is timedependent. Work from our group and others suggest particularly disappointing responses in survivors who begin swallowing therapy more than 2 years after completing curative RT. ${ }^{18} 19$ For instance, in the investigators' published case series of late-RAD, oral diet scores were observed to significantly deteriorate over a median follow-up of 10 months in the late post-RT period, despite standard swallowing exercise therapies with or without oesophageal dilation. Likewise, in secondary analysis of a National Cancer Institute-funded multi-site swallowing therapy trial among 117 survivors with chronic and late $\mathrm{RAD}$, response to swallowing therapy was time-dependent. QOL and diet scores improved most among those who started therapy $<1$ year after RT, with little improvement evident among those who started therapy more than 2 years post-RT. ${ }^{20}$

Clinical experience supports that survivors with late-RAD almost universally present with palpable, high-grade cervical fibrosis. Fibrosis is thought to compress peripheral nerve tracts, thereby contributing to denervation of critical swallowing muscles. ${ }^{21}$ Largely considered irreversible and potentially progressive, these normal tissue changes disrupt the intricate sensorimotor processes required to simultaneously close the airway, open the oesophagus and push a bolus through the pharynx for successful swallowing. With mature fibrosis, late-RAD coexists with other problems including impaired cervical range of motion (CROM) and abnormal cervical posture. ${ }^{22}$ Broad manifestations of radiation injury have been referred to as radiation fibrosis syndrome (RFS) with progressive myelo-radiculo-plexo-neuro-myopathic changes resulting in a host of functional challenges including cervicalgia and head drop syndrome. ${ }^{23}$ A recent cross-sectional analysis of musculoskeletal impairment in 29 long-term HNC survivors reported $89 \%$ had abnormal cervical posture with significant deviation in cervical extension relative to normative ranges ( $\mathrm{z}$-score: $0.63, \mathrm{p}<0.001$ ). Postural and CROM impairments significantly correlated with patientreported outcome (PRO) measures of shoulder and jaw function, but swallowing associations were not reported. ${ }^{22}$ There are, however, emerging data to support a correlation between cervical biomechanics and swallowing. Better CROM and skin pliability (as a clinical marker of cervical fibrosis) associated significantly with swallowing safety per Penetration-Aspiration Scale scores from videofluoroscopic swallowing evaluations in survivors with chronic RAD, however, in this secondary analysis of clinical trial data, change in cervical ROM or pliability did not associate with change in swallowing function after swallowing therapy. ${ }^{24}$

In long-term HNC survivors with late-RAD, circumferential cervical muscles can demonstrate reduced capacity for contraction and appropriate ROM due to RFS, which can result in a head drop position. Anterior and lateral cervical muscles are typically shortened and firm to touch due to progressive fibrotic tissue sclerosis. As a result of fibrotic cervical flexors, cervical extensors are overstretched and can become weak and atrophied over time. ${ }^{2325}$

Cervical posture is empirically a critical background factor facilitating safe swallowing. That is, when swallowing in a head drop position, the path of least resistance for any residual bolus in the pharynx is down into the airway. This might contribute to gravity-assisted aspiration (GASP), whereby post-swallow residue enters the airway more easily when the resting head posture is dropped. Indeed, GASP is a regular clinical observation in the investigators' practice. In contrast, with more normal upright cervical posture, residual bolus may dwell posteriorly in the pharynx waiting to be safely cleared into the oesophagus on a subsequent swallow as shown as proof of concept in exemplar case in figure 1.

Adjusting the swallow environment by improving cervical posture, as shown in figure 1 , is often overlooked as a therapeutic target for late-RAD. For these reasons and clinically recognising the high prevalence of forward head drop co-occurring with late-RAD, neutralising cervical posture by improving upper and lower cervical extension is the initial goal in the proposed MT 


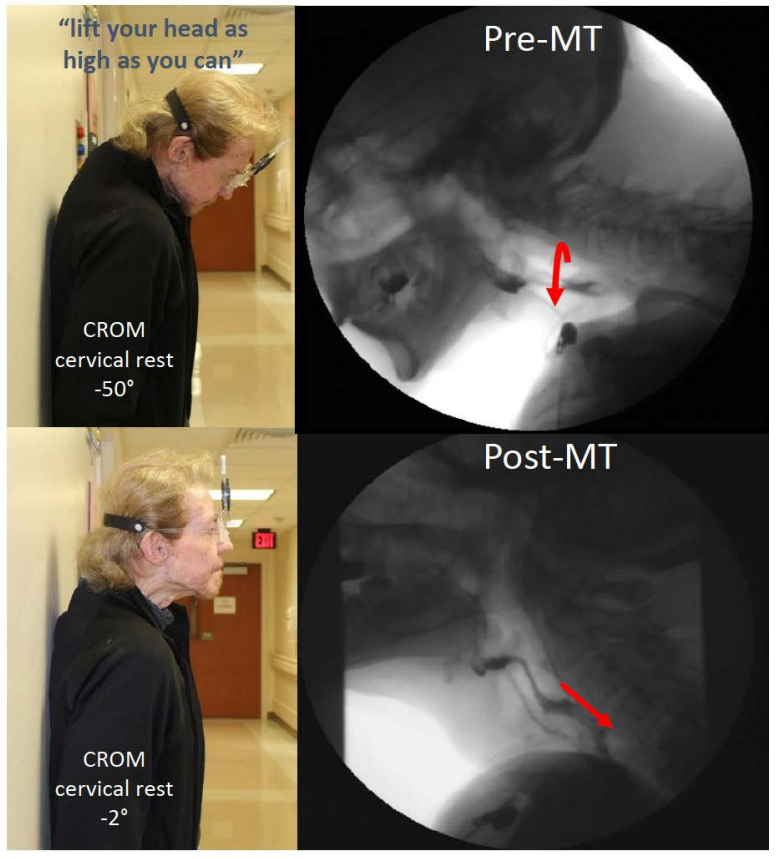

Figure 1 Cervical extension and aspiration improved in case example after manual therapy (MT). Exemplar case before (top) and after (bottom) single session of MT 18 years post-treatment, surgery and radiotherapy for head and neck cancer. Note red arrows on modified barium swallow study depicting residual bolus in pharynx directed anteriorly toward airway with cervical posture in resting forward head drop (top), and directed posteriorly toward oesophagus with cervical extension improved (bottom). While neither swallowing function or nor cervical biomechanics is normalised or ideal, functional gains were observed. CROM, cervical range of motion.

programme in this trial. Integration of this goal focused on priming or optimising the swallow environment prior to mobilising intrinsic swallowing musculature represents a novel element of our proposed MT swallow therapy programme, called MANTLE (Manual Therapy for Fibrosis-Related Late Effect Dysphagia).

Preliminary unpublished clinical data from the investigators detect an average $11^{\circ}$ improvement in a fibrosisrelated endpoint of cervical extension after a single session of MT $(\mathrm{p}<0.001)$, and notable qualitative remarks about functional gains after MT in clinical practice (eg, 'that's the first time I've felt myself swallow in years'). These early observations helped motivate the development of the MANTLE therapy programme and this trial. Acknowledging the typically progressive and refractory nature of late fibrotic effects, it is critical to understand the durability of improved CROM and whether this translates to better swallowing function.

\section{Objectives}

Therefore, the pilot MANTLE trial proposes to study a novel, adjunctive MT programme in patients with fibrosisrelated late-RAD with the following objectives:

1. To determine the feasibility and safety of MANTLE as a programme of treatment for fibrosis-related dysphagia in HNC survivors.

2. To estimate effect size, dose-response (number of treatment sessions to normalised CROM) and durability of MANTLE for improving CROM in HNC survivors with fibrosis-related late dysphagia.

3. To examine functional outcomes after MANTLE in HNC survivors with fibrosis-related late effects and their association with change in dysphagia grade, cervical extension and other cofactors.

\section{METHODS}

\section{Study design}

MANTLE is a single-institution, prospective single-arm unblinded pilot trial of MT in patients with late dysphagia after head and neck (HN) RT. Clinical schedules in the Section of Speech Pathology and Audiology at the University of Texas MD Anderson Cancer Center (MDACC) (Houston, Texas, USA) are screened to identify eligible patients referred for post-radiation swallow assessment. The investigators will enroll consecutive patients who meet eligibility and give written informed consent. Target enrolment is 24 participants who start the MANTLE programme, with up to 32 participants enrolled during screening. The first participant enrolled on 6 August 2018; trial completion is projected to occur in April 2021. MT is delivered according to a standard protocol for 6 weeks followed by a 6 -week washout period. Feasibility and safety will be examined. Serial assessments also include CROM, imaging and PROs. The trial schema is depicted in figure 2.

The study will be conducted according to the Declaration of Helsinki, Protection of Human Volunteers (21 CFR 50), Institutional Review Boards (21 CFR 56) and Obligations of Clinical Investigators (21 CFR 312). The principles of informed consent in the current edition of the Declaration of Helsinki will be implemented before any protocol-specific procedures or interactions are carried out. Informed consent will be obtained, in accordance with 21 CFR 50.25. The written consent document will

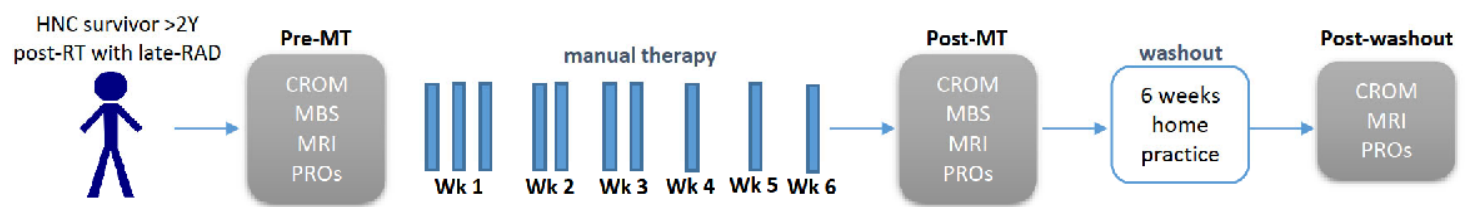

Figure 2 Manual Therapy for Fibrosis-Related Late Effect Dysphagia trial schema. CROM, cervical range of motion; HNC, head and neck cancer; MBS, modified barium swallow; MT, manual therapy; PROs, patient-reported outcomes; RAD, radiationassociated dysphagia; RT, radiotherapy. 
embody the elements of informed consent, as described in the Declaration of Helsinki and will also comply with local regulations of the Institutional Review Board (IRB) of MDACC.

Protocol amendments impacting eligibility, outcome and/or analysis are submitted for IRB approval, communicated through the institutional electronic protocol system to all relevant investigators, and updated in trial registration.

\section{Inclusion criteria}

1. Age $\geq 18$ years.

2. Late Dynamic Imaging Grade for Swallowing Toxicity (DIGEST) grade $\geq 2$ dysphagia on modified barium swallow (MBS) $\geq 2$ years after curative-intent radiotherapy for HNC.

3. Grade $\geq 2$ Common Terminology Criteria for Adverse Events (CTCAE) version 4.0 fibrosis.

4. Willing and able to return for 10 sessions that taper in frequency over 6 weeks of therapy.

\section{Exclusion criteria}

1. Active recurrent or second primary HN, central nervous system or thoracic cancer at the time of enrolment.

2. Active osteoradionecrosis or other non-healing wounds (eg, fistula, ulcer, soft tissue necrosis) in MT regions of interest at the time of enrollment.

3. History of subtotal or total glossectomy or total laryngectomy.

4. Functionally limiting cardiac, pulmonary, or neuromuscular disease.

5. Current tracheostomy.

\section{Treatment}

MT is commonly used in cancer survivors after RT and in the cervical region for pain and other indications. ${ }^{26-28}$ The proposed MT programme was developed by dysphagiaspecialised speech pathologists (SLP) dually licensed in massage therapy (HM) and certified in lymphoedema therapy (CLT) (HM, CP and KS) and summarised using the TIDieR template. ${ }^{29}$

The MANTLE programme added hierarchical goals for cervical biomechanics to the goals of a published swallowingfocused MT programme developed jointly by speech pathologists and an $\mathrm{HN}$ anatomist. ${ }^{26}$ MANTLE conceptualises and sequences MT and mobilisation targets from superficial to deep as detailed in table 1. First, targeting cervical extension to address the common 'head-drop' post-RT fibrotic cervical posture to a more natural, upright swallowing-optimised state (conjectured by the authors to be favourable for swallowing safety in the setting of pharyngeal residue) as shown in figure 1. Lateral flexion and cervical rotation are subsequently targeted to prime the tissues to access deeper swallowing regions-of-interest (ROIs) including the tongue, pharynx and larynx as summarised in table 1. Building on level I evidence from patients with non-cancer cervical pathology (ie, benign neck pain), the proposed MANTLE programme combines myofascial release and massage with range of motion (ROM) exercise to iteratively mobilise swallowing-related ROIs with a HEP for stretch and to initiate strengthening where stimulable. ${ }^{30-32}$ Table 1 displays the regions of interest where soft tissue mobilisation is hierarchically applied by the clinician during MT in addition to the functional goals and specific MT techniques. The list of exercises, duration and frequency in the HEP are also detailed in table 1.

In the MANTLE trial, an SLP (HM) who is a licensed massage and certified lymphoedema therapist (LMP and CLT) provided training to SLPs with CLT designations to perform the late-RAD MT programme. Patients are seen for 10 sessions over 6 weeks with titrating intensity (week 1: 3 sessions, weeks 2-3: 2 sessions weekly, weeks 4-6: 1 session weekly). The 10 sessions performed by the SLP in clinic include soft tissue mobilisation and instruction on a cervical stretching/strengthening home exercise programme (HEP); regions and sequence of targets for soft tissue mobilisation are detailed in table 1 . After the 10 sessions (over 6 weeks), the patient then transitions to a home practice of the HEP using a standardised clinical handout targeting cervical extension, bilateral cervical lateral flexion and bilateral cervical rotation without clinician assistance for a 6 -week washout period as detailed in table 1 .

\section{Subject registration}

All adult men and women scheduled for routine clinical MBS at MDACC are considered for participation in this study without regard to race, gender or socioeconomic status. Central registration is used for tracking study accrual and eligibility. Registration procedures include the following:

1. Interested patients are given an explanation of the study to include potential risks and benefits by the principal investigator (PI), coinvestigator or other designated medical personnel.

2. Investigator or other trained, designated personnel obtain signed informed consent before any study-specific procedures are performed.

3. The subject is seen and evaluated by the PI, coinvestigator or designee prior to enrolment. Screening assessments are performed.

4. The PI, coinvestigator or designee evaluate eligibility based on inclusion/exclusion criteria stated in this protocol.

\section{Screening evaluations}

Patients are evaluated by the speech pathologist and referring physician or advanced practice provider in the interdisciplinary $\mathrm{HNC}$ programme. Medical history, oral motor examination and oropharyngeal swallow function are recorded. The electronic medical record is reviewed for relevant history pertaining to eligibility criteria.

\section{Study evaluations}

Table 2 Summary of Treatment Evaluations.

Outcomes are assessed according to the schedule outlined in table 2. To facilitate retention, all 


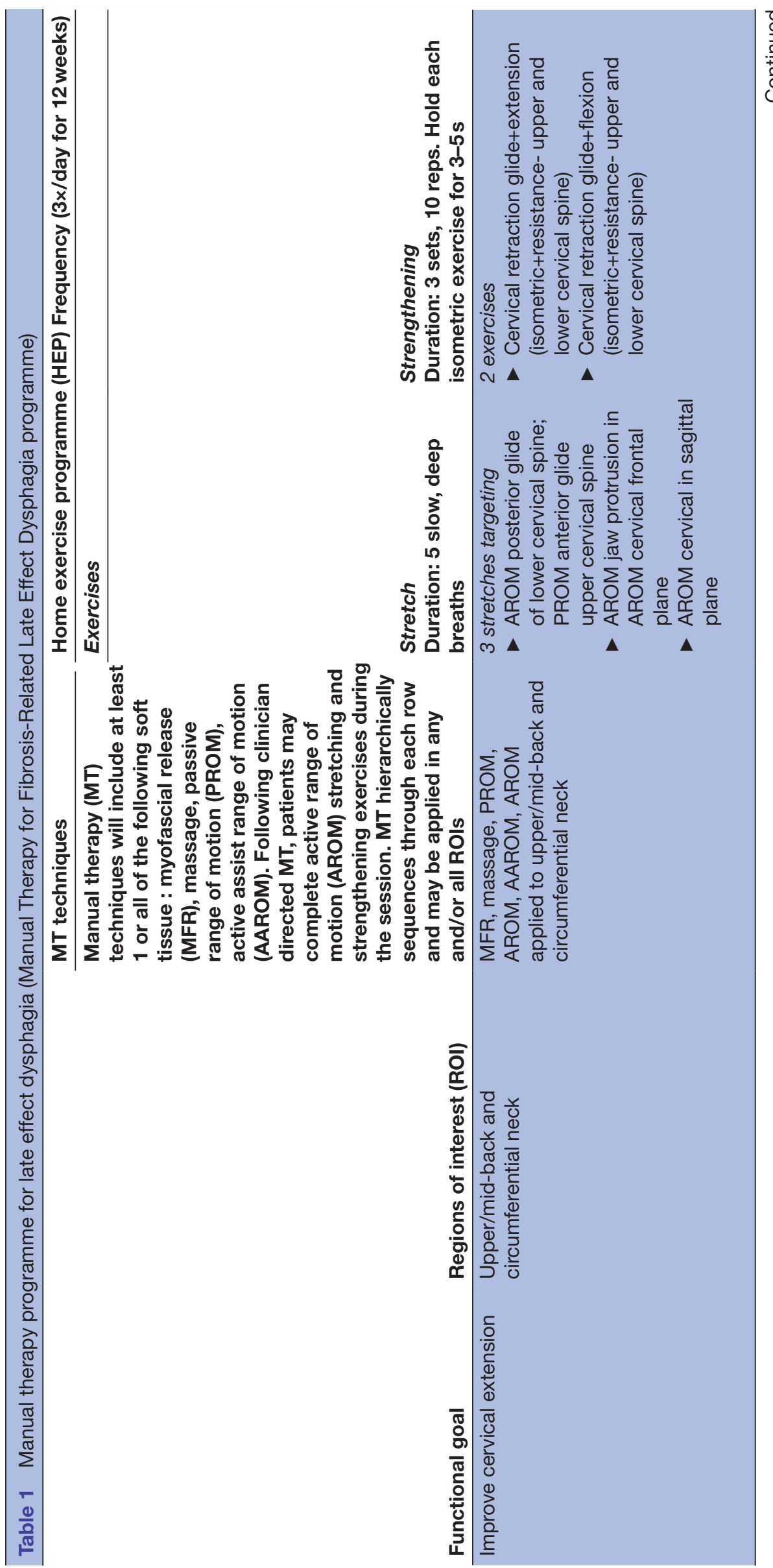




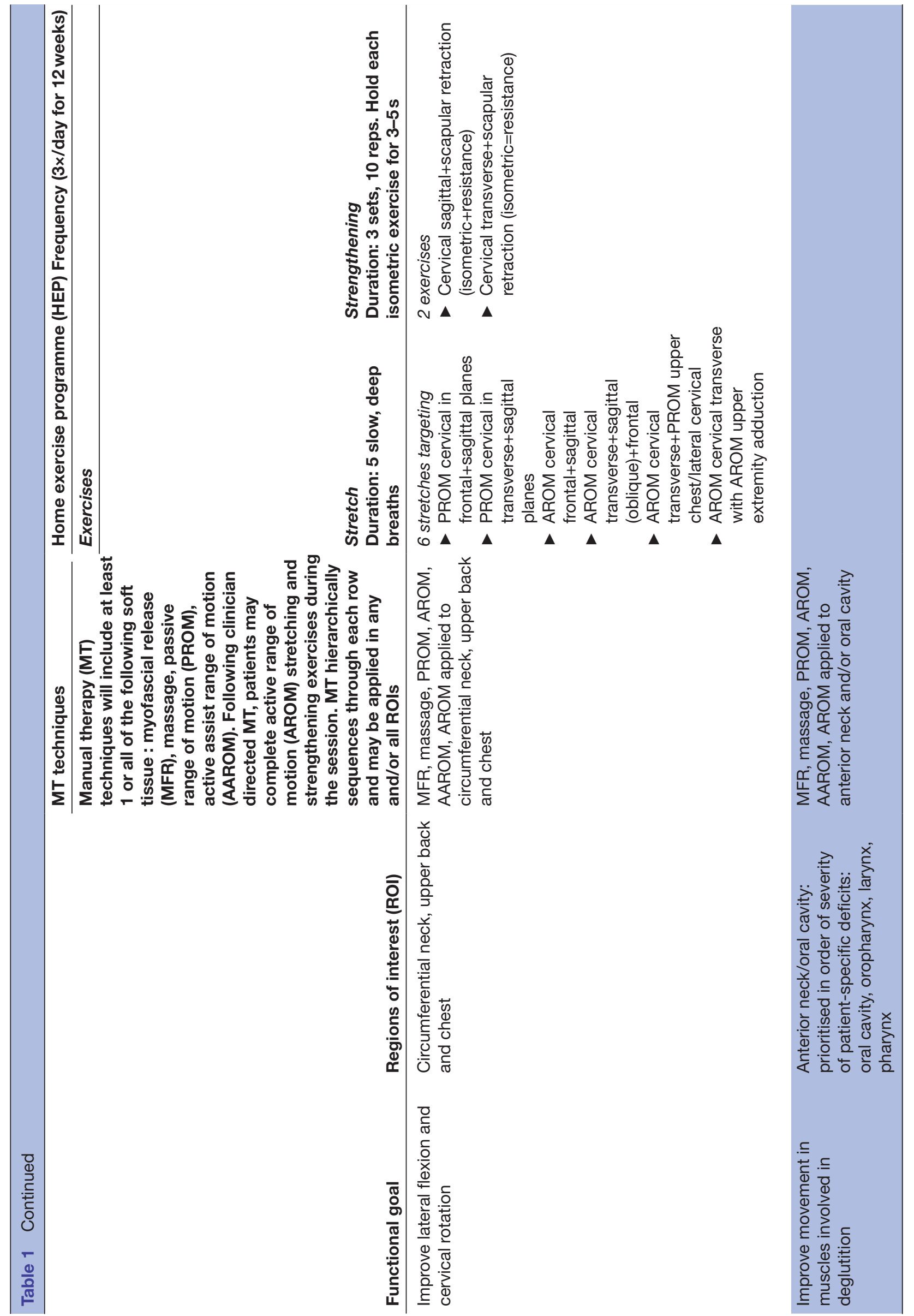




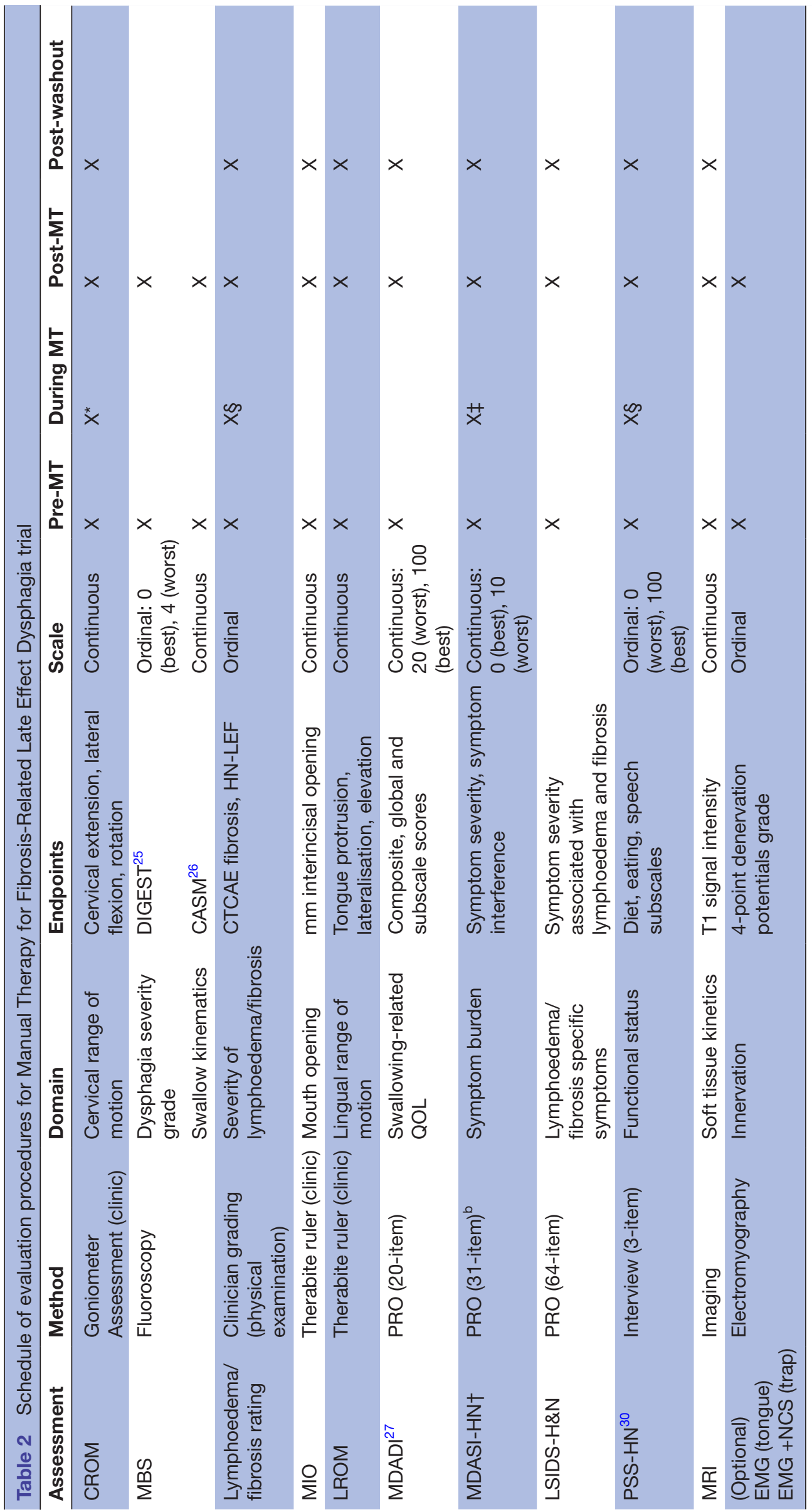

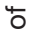

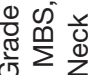

응

흘

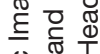

है훙

空变

$5 \sum_{0}$

虫

品

की

के के

एँ

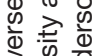

웅

혼

๘

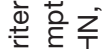

के $\frac{1}{0}$

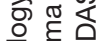

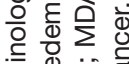

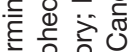

है

히를

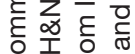

is 을 중

这的雳

응

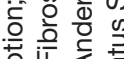

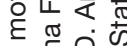

잉

बै 0 क

뜰 है

तु

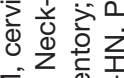

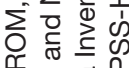

엉요 뜸

iิ $\frac{\pi}{1}$ 중 훌

क्ष

这文语

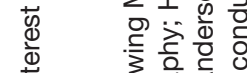

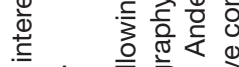

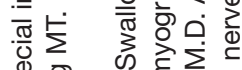

मे के के हे

刃

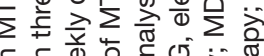

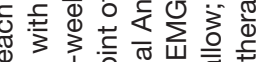

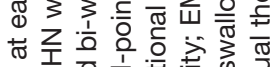

㪰

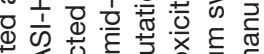

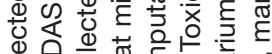

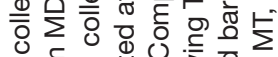

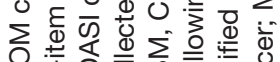

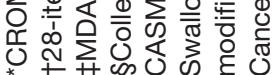


patient-completed questionnaires may be completed by paper or electronic means (ie, via REDCap), and data collection procedures are aligned with clinical visits. ${ }^{33}$ Outcome data will be collected from all participants; regardless of deviations from the therapy protocol. Details of data collection procedures follow.

CROM: patients are measured in an upright, seated position to reflect their natural cervical swallowing posture as the start position when CROM is measured. Clinic chairs are placed in a reproducible, fully upright standard position for every measurement. A goniometer measures CROM (degrees) to assess active cervical spine ROM. Five core CROM measures include cervical extension, sagittal plane at rest, lateral flexion (left/right), coronal plane at rest and lateral rotation (left/right). The primary CROM measure of interest is cervical extension. Cervical extension measures are highly reliable $(\mathrm{ICC}=0.90)$. Average extension measures in healthy adults aged 60-69 range from $57^{\circ}$ in men (SD: 10.5) to $65^{\circ}$ in women (SD: 13.3). Cervical extension measures decrease by approximately $5^{\circ}$ for each decade of life. ${ }^{35}$ Lingual and jaw ROM are also measured per published methods. ${ }^{36} 37$

Lymphoedema/fibrosis grading: clinician-grading of lymphoedema/fibrosis is conducted according to the published Head and Neck-Lymphedema Fibrosis, ${ }^{38}{ }^{39}$ CTCAE $^{40}$ and MD Anderson adaptation of the Foeldi lymphoedema rating, ${ }^{41}$ per physical examination of the patient. Clinical grading is a brief assessment that can be completed in $<15$ min using all three complementary sets of grading methods.

MBS studies: digital videos from clinical MBS will be scored by a trained speech pathologist blinded to the patient, study and follow-up interval using methods including the DIGEST ${ }^{42}$ and Computational Analysis of Swallowing Mechanics (CASM). ${ }^{434}$

MRI: multiparametric, serial MRI are acquired with a 1.5 T-3.0 T GE Discovery 750 MRI scanner (GE Healthcare, Wisconsin, USA) using laterally placed six-element flex coils centred on the base of tongue region. Feasibility and optimisation of this MRI paradigm have been described by the investigative team, ${ }^{45}$ with particular emphasis on immobilisation using dedicated H\&N coils and a flat insert table with an indexed base plate. Multiparametric imaging will be performed longitudinally
(pre-MT, post-MT and post-washout). Table 3 summarises candidate acquisitions for this study. Our primary candidate imaging biomarker of MANTLE-related soft-tissue change is normalised $\Delta \mathrm{T} 1_{\text {normalised }}$ signal intensity, as we have previously published this parameter's relevance as a dose-dependent soft tissue anatomic imaging biomarker of fibrosis. ${ }^{46}$ ROI delineation will be done using the investigators' published method and will include, among our published library of 72 normal structures, the following swallowing-relevant OARs: mylohyoid, geniohyoid and constrictors.

Optional intramuscular electromyography (EMG) and nerve conduction study (NCS) assess insertional activity in the tongue (XII nerve) as a marker of denervation. ${ }^{47}$ EMG recordings are conducted by a neurologist trained in clinical neurophysiology and denervation potentials graded per:

0 None.

1 Persistent, single trains of potentials in at least two areas.

2 Moderate number of potentials in three or more areas.

3 Many potentials in all areas.

4 Full interference pattern of potentials.

EMG and nerve conduction studies will also be taken in the trapezius muscle (XI nerve), as the region is easily accessible for non-invasive EMG with NCS and represents a muscle within the irradiated field with lower cranial nerve innervation. Both quantitative and qualitative EMG will be assessed. NCS will be assessed quantitatively, with waveforms generated from which we acquire amplitude, latency and conduction velocity. ${ }^{48}$ Optional EMG will not be conducted if platelets $<50 \times 10^{\wedge} 9 / \mathrm{L}$. Optional EMG and nerve conduction studies were added as a secondary procedure after trial activation.

M.D. Anderson Dysphagia Inventory (MDADI) is a written questionnaire to evaluate dysphagia-specific QOL in patients with $\mathrm{HNC}^{49}$ The 20-item MDADI questionnaire quantifies an individual's global, physical, emotional and functional perceptions of his or her swallowing ability. In an internal validation in 100 patients with HNC, concurrent validity was found to be moderate by comparison with the Performance Status Scale for Head and Neck Cancer Patients (PSS-HN)

Table 3 Candidate quantitative imaging parameters selected for Manual Therapy for Fibrosis-Related Late Effect Dysphagia trial

\begin{tabular}{lll}
\hline Acquisition type & Imaging parameter/biomarkers & Tissue injury correlate \\
\hline $\begin{array}{l}\text { T1-precontrast/ } \\
\text { postcontrast }\end{array}$ & T1 intensity, T1/R1, T1-rho & Radiation-associated fibrosis \\
T2/T2* map & T2 contrast, T2* & Radiation-associated oedema \\
DTI & Muscle fibre tractography and fractional anisotropy & $\begin{array}{l}\text { Muscle/nerve fibre/tumour microstructure, } \\
\text { directionality tracts }\end{array}$ \\
DCE & Perfusion parameters $\left(\mathrm{K}_{\text {trans }}, \mathrm{K}_{\mathrm{ep}}\right)$ & Tissue perfusion/microvessel permeability \\
\hline
\end{tabular}

DCE, dynamic contrast enhanced; DTI, diffusion tensor imaging. 
(Spearman correlation, 0.47-0.61). Correlation with the physical functional subscale (Spearman correlation, 0.40) and emotional subscale of 36-Item Short Form Survey (Spearman correlation, 0.36) demonstrated convergent and divergent validity, respectively, of the MDADI. Testretest reliability (physical, 0.86; emotional, 0.88; functional, 0.88 ) and internal consistency reliability (overall Cronbach's alpha, 0.96) were sound.

M.D. Anderson Symptom Inventory for Head and Neck Cancer $(M D A S I-H N)$ is a PRO questionnaire designed to measure severity or burden of systemic and HN-specific symptoms and their interference with or effect on patients' daily functioning. This 28-item multi-symptom inventory includes 13 core items ('systemic symptoms': pain, fatigue, sleep, etc), nine $\mathrm{HN}$-specific items ('local symptoms': dry mouth, mucus, shortness of breath, taste, etc) and six interference items (activity, work, relations, etc). The core MDASI items have been validated for use in populations of patients with cancer regardless of the specific diagnosis or type of therapy and thus can be used to compare overall burden of disease between different types of cancer. ${ }^{50}$ The $\mathrm{HN}$-specific items were validated internally with regard to construct and concurrent validity in patients with HNC. ${ }^{51}{ }^{52}$ Internal consistency reliability is high in the core, $\mathrm{HN}$-specific and interference items (Cronbach's alphas of 0.72-0.92). Validated linguistic translations (Chinese, French, German, Greek, Italian, Spanish and Turkish) of the MDASI-HN may be administered to non-English speaking participants.

Lymphedema Symptom Intensity and Distress Survey-Head and Neck (LSIDS-HE्EN) $)^{53}$ is a 64-item instrument designed to assess lymphoedema symptoms in patients with HNC. Survey items were selected to address six domains (HN-specific functioning, systemic symptoms, psychosocial issues, altered sensation symptoms, neck-shoulder musculoskeletal/skin symptoms and miscellaneous symptoms) identified by an expert panel. Preliminary testing of LSIDS-H\&N demonstrated both feasibility and readability.

$P S S-H N$ is a clinician-rated instrument rated by a semi-structured interview consisting of three questions: normalcy of diet, public eating and understandability of speech. ${ }^{54}$ The PSS-HN has been psychometrically validated and recommended by the National Comprehensive Cancer Network for measurement of swallowing and speech performance in patients with HNC.

Adherence logs: standard clinical adherence logs are given to track adherence to the HEP performed throughout the entire MANTLE trial. Patients are asked to complete paper logs with checkboxes to count completion of each HEP component on a daily basis. Logs are returned and reviewed at live MT sessions in attempt to validate responses or clarify ambiguity.

\section{Follow-up intervals}

Post-MT and post-washout evaluations (specified in table 2) will be conducted immediately after the 6 -week training period with an allowable window of 2 weeks.

\section{Safety and feasibility}

Safety and feasibility of MT: feasibility of MANTLE will be assessed by estimating programme completion rates, sources of attrition and adherence (ie, clinical attendance and adherence to HEP). Process evaluation checklists will be completed after each session to examine fidelity to the MANTLE treatment programme, and patients will be asked to log their home practice using a studyspecific adherence form. Adverse events (AEs) are also assessed and recorded. Cervical MT is a safe therapy in many populations. Risk of serious AEs is estimated to be 6 in 10 million. ${ }^{41}{ }^{42}$ Serious AEs (SAEs) relate to cardiovascular risk and are more common when providing a thrust manipulation technique that is employed in this protocol. Mild AEs, while still rare (estimated 1\%-2\% of patients), are more common and can include local discomfort, headache, lightheadedness, falls and fatigue. SAEs were not encountered in our preliminary retrospective review of patients receiving MT.

\section{Data management}

The data are maintained in an institutionally approved electronic data capture system with an integrated codebook. Data management adheres to institutional guidelines and policies for maintaining confidentiality to protect protected health information (PHI) from public viewing by safeguarding storage and disposal of documents containing PHI and computer workstations and databases that access PHI. Data validation will include missing data reports range checks for data values, and logical checks for plausible relationships. The PI, statistician and data manager will have access to the final trial dataset.

\section{Monitoring}

The trial will be monitored by the Office of Protocol Research at the MDACC subject to independent audit by MD Anderson's Internal Audit Department in accordance with the Texas Internal Audit Act and the University of Texas System Board of Regents and the Internal Audity Activity Charter. AEs will be recorded by the study team. The IRB will be notified of any related grade three or greater AEs and provided data to permit a safety review of the study treatment. The IRB may request additional meetings or safety reports as deemed necessary.

\section{Patient and public involvement}

The investigators did not formally engage a patient or public stakeholder team in trial development or recruitment strategies. Study data will be disseminated via peerreviewed publication made publicly available through PubMed Central, and shared with participants through this medium.

\section{Ethics and dissemination}

The research protocol and informed consent document was approved by the Institutional Review Board at the University of Texas MDACC. Findings will be disseminated through peer-reviewed publication that will be 
made publicly available on PubMed Central on acceptance for publication, in compliance with NIH public access policy.

\section{Statistical considerations}

\section{Statistical analysis and sample size justification}

The primary objective of MANTLE is to determine feasibility based on the programme completion rate. Investigators planned to enrol 24 patients and estimated an attrition rate of $20 \%$ to achieve a final sample size of 19 participants. Programme completion rate is estimated from the participants who start the MANTLE programme after screening procedures excluding screen failures and those who withdrawal before therapy starts. Note, the final protocol was amended to account for unanticipated prolonged treatment interruption or delay due to the institutional suspension of live clinical services and clinical research in response to the COVID-19 pandemic. For that reason, investigators increased maximum accrual to 32 participants and will cease enrolment after 24 participants start treatment without any COVID-19-related interruptions. The primary analysis will include the 24 participants who started MT and who did not experience the COVID-19-related interruption of study participation. Any study withdrawals or interruptions in study participation for reasons other than COVID-19 interruptions will be part of this feasibility analysis. In addition, as a sensitivity analysis, all available data, including any data collected from the participants interrupted by the COVID-19 research suspension, will be analysed in a stratified manner. Stratification by COVID-19 treatment interruptions was added to the protocol after activation in response to the unanticipated study impact of the pandemic.

For analysis of the primary objective to determine the feasibility and safety of the MANTLE programme, a completion rate of $75 \%$ will be considered the benchmark of feasibility. Completion rate will be defined by completion of the 6-week clinical MANTLE programme without withdrawing and attending a minimum of two sessions plus the post-treatment assessment. Session attendance will be monitored separately to assess adherence and fidelity. We will summarise fidelity and adherence to the standard MANTLE protocol using quantitative and qualitative methods. AEs will be tabulated.

For analysis of the secondary objective, we chose cervical extension as the primary CROM measure of interest and as the direct treatment target due to our preliminary data suggesting significant improvements after MT in patients with fibrosis-related late effects (one session mean $\Delta: 11 \%$; $\mathrm{p}<0.001$ ) as well as our conjectured relevance of CROM to swallowing safety. Cervical extension CROM measures are also highly reproducible $(\mathrm{ICC}=0.90)$ and are taken each standard MT session as part of routine clinical appointments to direct therapy. For this analysis, we will use simple descriptive statistics to first summarise baseline, post-MT1 and post-MT2 (after the 6-week washout period of exclusive home therapy) CROM measures for each anatomic plane. We will compare baseline to post-MT1 CROM measures using a one-sided paired t-test. With 24 patients and conservatively estimating an attrition rate of $20 \%$ based on our prior experience, the final sample size of 19 patients will provide $80 \%$ power to detect an effect size of $\mathrm{d}=0.593$, which corresponds to a $\triangle \mathrm{CROM}_{\text {baseline-post-MT }}$ of $10.68^{\circ}$ (assuming the $\mathrm{SD}=18$ per our pilot data in $\mathrm{HNC}$ ). To determine whether cervical extension normalises within 10 sessions of clinician-administered MT, post-MT CROM raw scores will be converted to z-scores based on age-specific and sex-specific norms to estimate the proportion of participants with post-MT cervical extension scores that fall within 2 SDs of normative values. To examine the durability of response, we will normalise post-MT2 CROM measures to determine the percentage of participants who maintain or improve (z-score $\geq$ post-MT score -2 SDs) post-wash-out. For the expected proportion of $80 \%$ who maintain or improve CROM, the $95 \%$ CI will extend $15 \%$ from the observed proportion. The number of MT sessions before normalisation of CROM will be tabulated. In exploratory analysis, we will plot CROM measures across time and will consider using linear mixed models to account for repeated outcome measures with adjustment for clinicodemographic covariates.

For the analysis of our tertiary objective, we will conduct a stepwise multivariable analysis to explore covariant swallow morphometrics associated with change in dysphagia grade and cervical extension after MT using the published CASM method. Swallow coordinates from all frames of pre-MT and post-MT MBS studies and covariates (time, bolus type, DIGEST ${ }_{\text {pre }}, \triangle$ DIGEST, age, sex, tumour site, surgery, $\triangle \mathrm{CROM}, \mathrm{CROM}_{\text {post }}$ ) will be specified in MorphoJ, an integrated morphometric software program, for stepwise analysis: (i) canonical variate analysis (CVA) to identify and rank covariates associated with swallow morphometric changes in patients improved/stable dysphagia ( $\triangle$ DIGEST), (ii) post hoc discriminant function analysis will be conducted next to visualise treatment-specific eigenvectors of swallowing muscle motion differences by covariates of interest from CVA (eg, pre-MT and post-MT) and (iii) morphometric regression to estimate post-RT eigenvectors associated with change in swallow severity per DIGEST and pre-MT and post-MT conditions. For CVA of $\mathrm{k}$ variables (12 coordinates motion) in G groups (DIGEST, MT), the total sample size must be larger than $[(2 \mathrm{k}-4)+(\mathrm{G}-1)]{ }^{55}$ requiring 21 patients.

\section{Exploratory analyses}

Secondary endpoints of MDADI, MDASI-HN, lingual and jaw ROM, lymphoedema/fibrosis staging, lingual and jaw ROM, PSS-HN and MRI parameters will be assessed according to their distributions (continuous: paired t-test or non-parametric Wilcoxon signed-rank test, ordinal: rank-invariant). Effect sizes, such as Cohen's d, with 95\% CIs will be calculated for each endpoint and interpreted. Exploratory analyses and correlative questions will be considered for hypothesis-generating purposes only. 


\section{DISCUSSION}

The highly focused long-term objective of the MANTLE trial is to improve swallowing function in some of the worst dysphagia presentations in HNC survivorship-that is, those with severe late-RAD we have shown to be often treatment refractory. The focus on late-RAD represents a departure from most therapy trials for RAD that suffer from population heterogeneity as a consequence of enrolling both early and late-RAD patients jointly, where the pathophysiology and trajectory of dysphagia almost certainly differs. By explicitly studying MT effects solely in late ( $>2$ years) post-radiated survivors, this line of research offers specificity of target in examining the therapeutic potential of this commonly used, but rarely studied treatment modality. The endpoints are thoughtfully constructed to estimate effect sizes of various avenues of clinical benefit including mobility, functional change, QOL and physical swallowing change. Any functional benefit for those with late-RAD could be meaningful as this represents a high burden, growing survivor population with disappointingly limited therapy options in current practice.

MANTLE was designed as a pilot study because we are trialling a novel therapy protocol intended for expansion to larger, confirmatory trials of efficacy or effectiveness in our programme of research on radiation-associated dysphagia. With the results of this pilot investigation, we expect to demonstrate that the novel MANTLE programme is feasible and safe to examine in a larger programme of research. Furthermore, we expect to estimate effect sizes achieved in secondary endpoints that will be necessary for power calculations in future trials of efficacy or effectiveness. The diverse outcomes panel was selected specifically to understand which data collection procedures (ie, functional measures, questionnaires, imaging) may be sensitive to possible changes with MT and merit inclusion in future, larger studies.

\section{Strengths and limitations}

The ideal dosing of MT for this indication is unknown. The dosing schedule is based on prior clinical experience as well as the MT evidence base. Published cervical MT programmes vary in intensity from $4+$ sessions over 2-7 weeks in populations with neck/shoulder dysfunction ${ }^{30-32}$ to 12 sessions over 4 weeks in non-HNC populations with fibrosis-related late effects in other body regions. ${ }^{56}$ The MANTLE programme is designed with 10 sessions over 6 weeks of clinician-directed MT simultaneous with the implementation of cervical HEP. Relative to other cervical MT programmes in the literature, this represents a fairly intense MT schedule to match the known pathophysiology and duration of injury of the target population with late-RAD. The MANTLE therapy schedule is intentionally titrated using a scaffold approach in therapy schedule to offer more frequent upfront soft tissue manipulation while transitioning the patient to the independence of a HEP for maintenance. The investigators acknowledge that the therapy schedule developed for the MANTLE protocol may require further refinement as the results mature; however, 10 sessions over 6 weeks were judged by the investigators to represent a time interval during which therapy response should be detectable. Future directions of this trial might include adjusting the dosing of MT and HEP to achieve similar results.

The aims and outcome measures are thoughtfully constructed to provide pilot data regarding the feasibility/ safety (aim 1), dose and durability (aim 2) and functional translation (aim 3) of MANTLE as an adjunctive therapeutic modality for late-RAD. On completion of aim 1 , we expect to show a therapy completion rate of $75 \%$ as a marker of feasibility. For aim 2, we expect to demonstrate that CROM can improve within 10 sessions of MANTLE in at least $80 \%$ of HNC survivors with late-RAD. Aim 3 will provide effect sizes estimates of swallowing function changes after MANTLE. In this pilot trial, we expect to observe attrition $<25 \%$, adherence $>60 \%$, no therapyrelated grade $\geq 3 \mathrm{AEs}$ and sufficient power to estimate Cohen's $d$ effect size $\geq 0.50$ for the primary secondary endpoint of interest CROM.

Cervical measurements are challenging to obtain in the late-RAD population when severe cervical postural abnormalities are present. In order to achieve a more neutral or upright head position, cervical extension is required in the upper cervical, lower cervical and upper thoracic spine. Due to the severity of head drop in the pilot data, we were unable to measure upper cervical movement in isolation to capture degree of head drop or forward head posture (FHP). Measurements were out of range and could not be obtained with a traditional or Q-ROM computer-generated goniometer due to the degree of lower cervical flexion. Valid and reliable tools to measure FHP in other populations such as craniovertebral angle measures merit exploration in future research in HNC survivors. ${ }^{57}$ The omission of such postural measures and upper cervical extension/dorsal glide as an evaluation measurement is a limitation; it was recognised and accounted for by implementing dedicated stretching and strengthening exercises to target upper cervical spinal movements to improve FHP, if present.

Exploring functional endpoints of the therapeutic trial, we expect to also evaluate the mechanism of functional change in swallowing (per DIGEST), muscle motion parameters (per CASM) associated with functional swallowing improvements (per DIGEST) using radiographic MBS studies. The post-MANTLE MBS is conducted immediately following 6 weeks of MT. Even healthy individuals may require 8-12 weeks to see functional improvement with stretching and strengthening. ${ }^{58}{ }^{59}$ As such, the post-MANTLE MBS after just 6 weeks of therapy may be earlier than maximal benefit is achieved. Nonetheless, at 6 weeks, any change detected on MBS could be more directly attributed to the MANTLE therapy prior to the washout period and may reflect stimulability in the tissue.

The final data collection is 12 weeks after starting MANTLE. While long-term follow-up of dysphagia progression after MANTLE is not feasible in the timeframe of this pilot trial, any positive changes in CROM, 
PROs, soft tissue (per MRI), or radiographic or perceived swallowing function is likely meaningful because late-RAD is currently considered a treatment refractory toxicity syndrome.

\section{Future directions}

If proven safe and feasible, future studies will need to address/investigate efficacy, effectiveness, sustainability of therapeutic gains, ideal schedules, frequency and combination of MT techniques, and best matching of mobility focused MT with direct functional therapies. For instance, might MANTLE prime the patient with late-RAD to achieve better functional gains during a bolus driven paradigm like the McNeil Dysphagia Therapy Programme $?^{60}$ Future considerations should also include remote practice as it is rapidly expanding in the era of the COVID-19 pandemic. With this in mind, it will become even more important to understand the outcome of the cervical HEP alone (without soft tissue manipulation as it is used in MANTLE) among patients with RAD who may not be able to access in-person clinical services for soft tissue manipulation. We believe that the proposed MANTLE trial could provide pilot data that might justify practice-changing clinical trials for the growing number of HNC survivors who have no proven options to manage late-RAD.

\section{Author affiliations}

${ }^{1}$ Head and Neck Surgery, Radiation Oncology, University of Texas MD Anderson Cancer Center, Houston, Texas, USA

${ }^{2} \mathrm{Head}$ and Neck Surgery, University of Texas MD Anderson Cancer Center, Houston, Texas, USA

${ }^{3}$ Biostatics, University of Texas MD Anderson Cancer Center, Houston, Texas, USA ${ }^{4}$ Radiation Physics, University of Texas MD Anderson Cancer Center, Houston, Texas, USA

${ }^{5}$ Neurology, University of Texas MD Anderson Cancer Center, Houston, Texas, USA ${ }^{6}$ Radiation Oncology, University of Texas MD Anderson Cancer Center, Houston, Texas, USA

Acknowledgements The authors gratefully acknowledge clinical contributions from the Section of Speech Pathology and Audiology, administrative support from Ms Angela Kurtz, and protocol administration by the Clinical Research Group in the Department of Head and Neck Surgery.

Contributors All listed coauthors read and approved the manuscript, and performed the following: substantial contributions to the conception or design of the work; or the acquisition, analysis, or interpretation of trial concept and data; drafting the work or revising it critically for important intellectual content; final approval of the version to be published; agreement to be accountable for all aspects of the work in ensuring that questions related to the accuracy or integrity of any part of the work are appropriately investigated and resolved. Specific individual contributions in addition to all criteria above are listed as follows: KH: principal investigator; corresponding/primary author; conceived, coordinated and directed all study activities, responsible for data collection, project integrity, manuscript content and editorial oversight and correspondence; direct oversight of study personnel. HM: manual therapy lead; developed MANTLE therapy programme; trained MANTLE clinicians. SB: operationalisation and refinement of data collection procedure, primary trial manager. $\mathrm{KH}, \mathrm{HM}, \mathrm{CP}, \mathrm{KS}, \mathrm{KW}$, SYL, CF: direct patient care provision, direct outcomes assessment and development and refinement of clinical data collection procedures. CW: developed statistical analysis plan. CF, JW: responsible for programmatic and infrastructure oversight for MRI analysis and DICOM segmentation; direct and final oversight of MRI data collection; direct oversight of trainee personnel. SL: direct and final oversight of surgical data collection. KW: direct and final oversight of EMG data collection.
Funding This work is supported by the National Institutes of Health (NIH)/National Cancer Institute (NCI) 5R21CA226200. The content is solely the responsibility of the authors and does not necessarily reflect the official views of the National Institutes of Health.

Competing interests None declared.

Patient and public involvement Patients and/or the public were not involved in the design, or conduct, or reporting, or dissemination plans of this research.

Patient consent for publication Not required.

Provenance and peer review Not commissioned; externally peer reviewed.

Open access This is an open access article distributed in accordance with the Creative Commons Attribution Non Commercial (CC BY-NC 4.0) license, which permits others to distribute, remix, adapt, build upon this work non-commercially, and license their derivative works on different terms, provided the original work is properly cited, appropriate credit is given, any changes made indicated, and the use is non-commercial. See: http://creativecommons.org/licenses/by-nc/4.0/.

ORCID iD

Katherine Hutcheson http://orcid.org/0000-0003-3710-5706

\section{REFERENCES}

1 Hunter KU. Toxicities affecting quality of life after chemo-IMRT of oropharyngeal cancer: prospective study of patient-reported, observer-rated, and objective outcomes. Int J Radiat Oncol Biol Phys 2013;85:935-40.

2 Eisbruch A, Kim HM, Feng FY, et al. Chemo-Imrt of oropharyngeal cancer aiming to reduce dysphagia: swallowing organs late complication probabilities and dosimetric correlates. Int J Radiat Oncol Biol Phys 2011;81:e93:9-99.

$3 \mathrm{Xu} \mathrm{B}$, Boero IJ, Hwang L, et al. Aspiration pneumonia after concurrent chemoradiotherapy for head and neck cancer. Cancer 2015;121:1303-11.

4 Chaturvedi AK, Engels EA, Pfeiffer RM, et al. Human papillomavirus and rising oropharyngeal cancer incidence in the United States. J Clin Oncol 2011;29:4294-301.

5 Awan MJ. Late radiation-associated dysphagia (late-RAD) with lower cranial neuropathy after oropharyngeal radiotherapy: a preliminary dosimetric comparison. Oral Oncol 2014;50:746-52.

6 M. D. Anderson Head Neck Cancer Symptom Working Group. Beyond mean pharyngeal constrictor dose for beam path toxicity in non-target swallowing muscles: Dose-volume correlates of chronic radiation-associated dysphagia (RAD) after oropharyngeal intensity modulated radiotherapy. Radiother Oncol 2016;118:304-14.

7 Kamal M, Mohamed ASR, Volpe S, et al. Radiotherapy dosevolume parameters predict videofluoroscopy-detected dysphagia per DIGEST after IMRT for oropharyngeal cancer: Results of a prospective registry. Radiother Oncol 2018;128:442-51.

8 Ang KK, Harris J, Wheeler R, et al. Human papillomavirus and survival of patients with oropharyngeal cancer. N Engl J Med 2010;363:24-35.

9 Fakhry C, Westra WH, Li S, et al. Improved survival of patients with human papillomavirus-positive head and neck squamous cell carcinoma in a prospective clinical trial. J Natl Cancer Inst 2008;100:261-9.

10 Weinberger PM, Yu Z, Haffty BG, et al. Molecular classification identifies a subset of human papillomavirus--associated oropharyngeal cancers with favorable prognosis. J Clin Oncol 2006;24:736-47.

11 Caudell JJ, Schaner PE, Meredith RF, et al. Factors associated with long-term dysphagia after definitive radiotherapy for locally advanced head-and-neck cancer. Int J Radiat Oncol Biol Phys 2009;73:410-5.

12 Hutcheson KA, Lewin JS, Barringer DA, et al. Late dysphagia after radiotherapy-based treatment of head and neck cancer. Cancer 2012;118:5793-9.

13 Awan MJ, Mohamed AS, Lewin JS, et al. Late radiation-associated dysphagia (late-RAD) with lower cranial neuropathy after oropharyngeal radiotherapy: a preliminary dosimetric comparison. Oral Oncol 2014;50:746-52.

14 Hutcheson KA. Late radiation-associated dysphagia (rad) in head and neck cancer survivors. Perspectives on Swallowing and Swallowing Disorders2013;22:61-72.

15 Hutcheson KA, Yuk M, Hubbard R, et al. Delayed lower cranial neuropathy after oropharyngeal intensity-modulated radiotherapy: A cohort analysis and literature review. Head Neck 2017;39:1516-23. 
16 Aggarwal P, Zaveri JS, Goepfert RP, et al. Swallowing-related outcomes associated with late lower cranial neuropathy in long-term oropharyngeal cancer survivors: cross-sectional survey analysis. Head Neck 2019;41:3880-94.

17 Rubin EB, Buehler AE, Halpern SD. States worse than death among hospitalized patients with serious illnesses. JAMA Intern Med 2016;176:1557-9.

18 Langmore SE, McCulloch TM, Krisciunas GP, et al. Efficacy of electrical stimulation and exercise for dysphagia in patients with head and neck cancer: a randomized clinical trial. Head Neck 2016;38:E1221:31-1231.

19 Hutcheson KA, Kelly S, Weber RS. Offering more for persistent dysphagia after head and neck cancer: The evolution of boot camp swallowing therapy. Boston, MA: Combined Otolaryngology Spring Meeting, 2015

20 Van Daele DJ, Langmore SE, Krisciunas GP, et al. The impact of time after radiation treatment on dysphagia in patients with head and neck cancer enrolled in a swallowing therapy program. Head Neck 2019;41:606-14.

21 Hutcheson KA, Yuk MM, Holsinger FC. Late radiation-associated dysphagia (late-RAD) with lower cranial neuropathy in long-term oropharyngeal cancer survivors: video case reports. Head Neck 2015;37:56-62.

22 Ghiam MK, Mannion K, Dietrich MS, et al. Assessment of musculoskeletal impairment in head and neck cancer patients. Support Care Cancer 2017;25:2085-92.

23 Stubblefield MD. Radiation fibrosis syndrome: neuromuscular and musculoskeletal complications in cancer survivors. PM\&R 2011;3:1041-54.

24 Silbergleit AK, Schultz L, Krisciunas G, et al. Association of neck range of motion and skin caliper measures on dysphagia outcomes in head and neck cancer and effects of neck stretches and swallowing exercises. Dysphagia 2020;35:360-8.

25 Rowin J, Cheng G, Lewis SL, et al. Late appearance of dropped head syndrome after radiotherapy for Hodgkin's disease. Muscle Nerve 2006;34:666-9.

26 Krisciunas GP, Golan H, Marinko LN, et al. A novel manual therapy programme during radiation therapy for head and neck cancer - our clinical experience with five patients. Clin Otolaryngol 2016;41:425-31.

27 Gross AR, Hoving JL, Haines TA, et al. A cochrane review of manipulation and mobilization for mechanical neck disorders. Spine 2004:29:1541-8.

28 Gross AR, Kay T, Hondras M, et al. Manual therapy for mechanical neck disorders: a systematic review. Man Ther 2002;7:131-49.

29 Hoffmann TC, Glasziou PP, Boutron I, et al. Better reporting of interventions: template for intervention description and replication (TIDieR) checklist and guide. BMJ 2014;348:g1687.

30 Page MJ, Green S, Kramer S, et al. Manual therapy and exercise for adhesive capsulitis (frozen shoulder). Cochrane Database Syst Rev 2014:Cd011275.

31 Camargo PR, Alburquerque-Sendin F, Avila MA, et al. Effects of Stretching and strengthening exercises, with and without manual therapy, on scapular kinematics, function, and pain in individuals with shoulder impingement: a randomized controlled trial. J Orthop Sports Phys Ther2015;45:984-97.

32 Trott CA, Ruiz Aguila ME, Leaver AM. The clinical significance of immediate symptom responses to manual therapy treatment for neck pain: observational secondary data analysis of a randomized trial. Man Ther 2014;19:549-54.

33 Harris PA, Taylor R, Thielke R, et al. Research electronic data capture (REDCap)-A metadata-driven methodology and workflow process for providing translational research informatics support. J Biomed Inform2009;42:377-81.

34 Harris PA, Taylor R, Minor BL, et al. The REDCap consortium: Building an international community of software platform partners. J Biomed Inform 2019;95:103208

35 Youdas JW, Garrett TR, Suman VJ, et al. Normal range of motion of the cervical spine: an initial goniometric study. Phys Ther 1992;72:770.

36 Dijkstra PU, Huisman PM, Roodenburg JL. Criteria for trismus in head and neck oncology. Int J Oral Maxillofac Surg 2006;35:337-42.

37 Lazarus CL, Husaini H, Jacobson AS, et al. Development of a new lingual range-of-motion assessment scale: normative data in surgically treated oral cancer patients. Dysphagia 2014:29:489-99.
38 Deng J, Ridner SH, Wells N, et al. Development and preliminary testing of head and neck cancer related external lymphedema and fibrosis assessment criteria. Eur J Oncol Nurs 2015;19:75-80.

39 Deng J, Dietrich MS, Ridner SH, et al. Preliminary evaluation of reliability and validity of head and neck external lymphedema and fibrosis assessment criteria. Eur J Oncol Nurs 2016;22:63-70.

40 Common Terminology Criteria for Adverse Events (CTCAE). v4.03 ED. Bethesda, MD: National Institutes of Health, National Cancer Institute, Department of Health and Human Services, 2010.

41 Lewin JS, Hutcheson KA, Barringer DA, et al. Preliminary experience with head and neck lymphedema and swallowing function in patients treated for head and neck cancer. Perspect Swal Swal Dis 2010;19:45-52.

42 Rosenbek JC, Robbins JA, Roecker EB, et al. A penetrationaspiration scale. Dysphagia 1996;11:93-8.

43 Schwertner RW, Garand KL, Pearson WG. A novel imaging analysis method for capturing pharyngeal constriction during swallowing. J Imaging Sci 2016;1. [Epub ahead of print: 2508 2016].

44 Taylor BK, Blair J, et al. Computational analysis of swallowing mechanics underlying impaired epiglottic inversion. Laryngoscope 2016;126:1854-8.

45 Ding Y, Mohamed ASR, Yang J, et al. Prospective observer and software-based assessment of magnetic resonance imaging quality in head and neck cancer: should standard positioning and immobilization be required for radiation therapy applications? Pract Radiat Oncol 2015;5:e299-308.

46 Messer JA, Mohamed AS, Hutcheson KA, et al. Magnetic resonance imaging of swallowing-related structures in nasopharyngeal carcinoma patients receiving IMRT: Longitudinal dose-response characterization of quantitative signal kinetics. Radiother Oncol 2016;118:315-22.

47 Perlman AL, Palmer PM, McCulloch TM, et al. Electromyographic activity from human laryngeal, pharyngeal, and submental muscles during swallowing. J Appl Physiol 1999;86:1663-9.

48 Preston DC, Shapiro BE. Electromyography and neuromuscular disorders: clinical-electrophysiologic correlations. 3rd ed. Philadelphia: Saunders, 2013.

49 Chen AY, Frankowski R, Bishop-Leone J, et al. The development and validation of a dysphagia-specific quality-of-life questionnaire for patients with head and neck cancer: the M. D. Anderson dysphagia inventory. Arch Otolaryngol Head Neck Surg 2001;127:870-6.

50 Cleeland CS, Mendoza TR, Wang XS, et al. Assessing symptom distress in cancer patients: the M.D. Anderson symptom inventory. Cancer 2000;89:1634-46.

51 Rosenthal DI, Mendoza TR, Chambers MS, et al. Measuring head and neck cancer symptom burden: the development and validation of the M. D. Anderson symptom inventory, head and neck module. Head Neck 2007;29:923-31.

52 Rosenthal DI, Mendoza TR, Fuller CD, et al. Patterns of symptom burden during radiotherapy or concurrent chemoradiotherapy for head and neck cancer: a prospective analysis using the University of Texas MD Anderson Cancer Center Symptom Inventory-Head and Neck Module. Cancer 2014:120:1975-84.

53 Deng J, Ridner SH, Murphy BA, et al. Preliminary development of a lymphedema symptom assessment scale for patients with head and neck cancer. Support Care Cancer 2012;20:1911-8.

54 List MA, Ritter-Sterr C, Lansky SB. A performance status scale for head and neck cancer patients. Cancer 1990;66:564-9.

55 Webster M, Sheets HD. A practical introduction to landmark-based geometric morphometrics. Paleontol. Soc. pap. 2010;16:163-88.

56 Cho Y, Do J, Jung S, et al. Effects of a physical therapy program combined with manual lymphatic drainage on shoulder function, quality of life, lymphedema incidence, and pain in breast cancer patients with axillary web syndrome following axillary dissection. Support Care Cancer 2016;24:2047-57.

57 Shaghayegh Fard B, Ahmadi A, Maroufi N, et al. Evaluation of forward head posture in sitting and standing positions. Eur Spine $J$ 2016;25:3577-82.

58 Jones DA, Rutherford OM. Human muscle strength training: the effects of three different regimens and the nature of the resultant changes. J Physiol 1987;391:1-11.

59 Seo MW, Jung HC, Song JK, et al. Effect of 8 weeks of pre-season training on body composition, physical fitness, anaerobic capacity, and isokinetic muscle strength in male and female collegiate taekwondo athletes. J Exerc Rehabil 2015;11:101-7.

60 Carnaby-Mann GD, Crary MA. McNeill dysphagia therapy program: a case-control study. Arch Phys Med Rehabil 2010;91:743-9. 